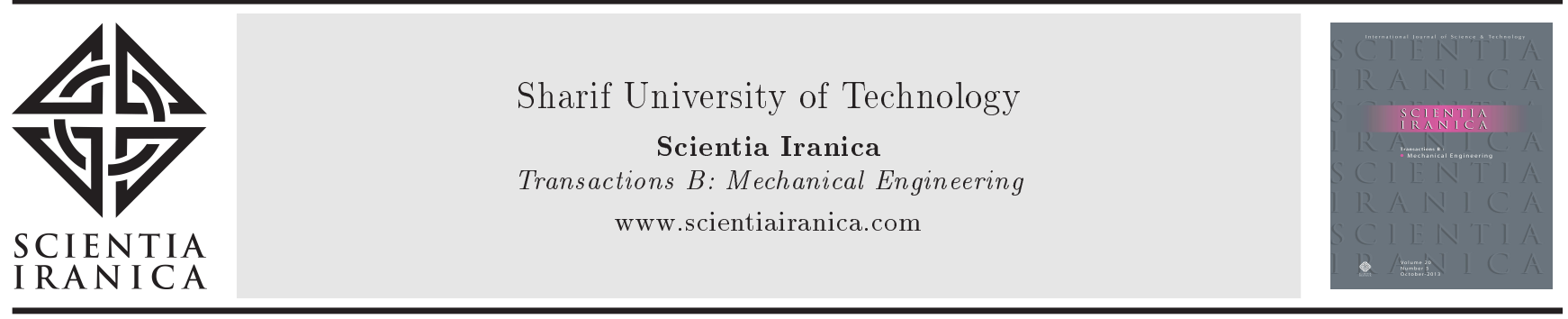

Research Note

\title{
Aerosol nano-particle charger simulations and optimizations
}

\author{
A. Shaygani ${ }^{\mathrm{a}}$, M.S. Saidi ${ }^{\mathrm{b}, *}$ and M. Sani ${ }^{\mathrm{a}}$ \\ a. School of Engineering and Science, Sharif University of Technology, Kish, Iran. \\ b. School of Mechanical Engineering, Sharif University of Technology, Tehran, P.O. Box 11155-9567, Iran. \\ Received 14 December 2012; received in revised form 5 August 2015; accepted 4 April 2016
}

\author{
KEYWORDS \\ Aerosol; \\ Charger; \\ Corona discharge; \\ CFD; \\ Nano; \\ Optimization.
}

\begin{abstract}
Size distribution of aerosol particles is prevalently obtained through electrical characterization techniques and study of charged particles' dynamics in the presence of electric field. In this work, a wire-cylinder corona charger is presented, redesigned, and aerodynamically optimized. An initial 2D axisymmetric geometry of the charger was employed for the simulations using the Computational Fluid Dynamics (CFD) commercial code FLUENT 6.3.26. Through successive attempts, a new geometry was obtained by streamlining the walls to eliminate the undesired vortices produced in the flow field of the previous ones. The process optimized the charger by minimizing losses and dilutions of the particles. For electrical simulations of the charger, a new numerical algorithm was designed based on the steady-state corona discharge to work with segregated solvers to satisfy governing equations. The algorithm was validated using a one-dimensional semianalytic solution of corona discharge. Tracing particles for the optimized geometry, the percentage of losses was calculated $6 \%$, whereas the loss in the old geometry was more than $30 \%$. The average charge and charge distributions induced on particles were also calculated with evaluation of the residence times in the charger.
\end{abstract}

(C) 2016 Sharif University of Technology. All rights reserved.

\section{Introduction}

Aerosol technology covers many research areas, including embracing environmental studies, contamination control and atmospheric research, energy and combustion studies, and filtering and powder coating Hinds [1]. The very first and critical step in nano-particle technologies is improving measurement capabilities. Particle size distribution is usually determined through the electrical mobility classifications, especially for particles smaller than 300 nanometers in diameter.

In order to classify aerosols according to elec-

*. Corresponding author. Tel.: +98 2166165558

E-mail addresses: shayganiafshin@gmail.com (A.

Shaygani); mssaidi@sharif.edu (M.S. Saidi);

msani@sharif.edu (M. Sani) trical mobility, sufficient amount of charge must be induced on each individual particle. Passing through electric field, their dynamics and motion reveal the size distribution. When charging mechanism occurs due to random collisions of ions and particles (Brownian motions) in the absence of very strong electric field, the process is called diffusion charging. This mechanism is dominant in smaller particles less than 200 nanometers in diameter. In the presence of strong electric field, charging by unipolar ions is called field charging (dominant in particles bigger than 200 nanometers) [2]. The only method that could be used to generate ions at high enough concentration is corona discharge. Many of the presented nano-particle chargers in the literature employ corona discharge [3]. Corona chargers are widely used in commercial aerosol classification instruments such as Differential Mobility Spectrometer and 
Electrical Aerosol Spectrometer [4]. The experimental studies on the performance of needle corona based chargers include Lin Li and Da-Ren Chen, [5], Qi et al. [6], and Park et al. [7]. On the wire corona chargers, Kruis and Fissan [8], and Alisoy et al. [9] provide numerical investigations. In the following, simulations and optimizations of the wire-cylinder corona charger are presented. Some reasons for choosing this type of charger in our study include:

1. This work was supposed to pave the way for the later experimental studies. Choosing a charger that could be easily manufactured was one of our priorities;

2. This charger, according to some studies (e.g., [10]), is a very efficient corona charger;

3. The less sophisticated geometry than that of the needle chargers made it possible to provide a onedimensional analytic solution and validate numerical calculations.

\section{Materials and methods}

\subsection{Description of aerosol nano-particle charger}

One of the aerosol chargers, known as Hewitt-type charger, that aided corona discharge was proposed by Biskos et al. [11]. As shown in Figure 1, it consists of two concentric cylinders with a central coronawire. Due to a high electric field between positive (or negative) corona-wire and grounded outer metal cylinder, ions are produced in the vicinity of the wire and migrate to the grounded electrode and flow into the charging zone through the central perforated cylinder. The zone between the two cylinders (charging zone) is the passage of aerosol flow. The inner perforated cylinder allows aerosol stream to flow in a laminar regime while preventing particles from interfering with ion generation area around the wire and being lost through flowing out with the sheath air. In the case that the inner perforated cylinder is conducting, it

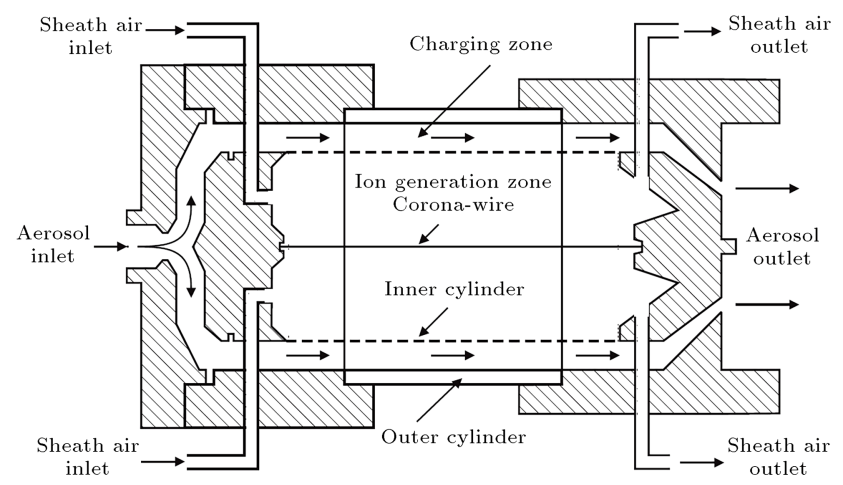

Figure 1. Schematic layout of the wire-cylinder charger; Biskos et al. [11]. serves as a grounded third electrode. If so, the outer electrode (outer cylinder) can be grounded as well, or powered by an $\mathrm{AC}$ voltage. The active charging region has a total length of $60 \mathrm{~mm}$. The diameters of wire and cylinders were $16 \mu, 50 \mathrm{~mm}$, and $74 \mathrm{~mm}$, respectively, and the aerosol flow rate was $5 \mathrm{lpm}$.

\subsection{Mathematical model}

\subsubsection{Governing electrical equations}

Corona occurs when a high enough potential difference is applied between at least two electrodes with different radii of curvatures, including dielectric insulator inbetween. The electrode with sharp edges or smaller radius of curvature is called corona electrode. The blunt electrode with much larger radius of curvature is usually grounded. Conservation of electric charge (current continuity) or charge transport equation combined with basic postulates of electrostatics describes the mathematical model of corona discharge by the following equations [12]:

$$
\begin{aligned}
& \nabla^{2} V=-\frac{\rho}{\varepsilon_{0}}, \\
& \nabla . J=-\frac{\partial \rho}{\partial t}+S .
\end{aligned}
$$

In the above equations, $V(\mathrm{~V})$ is the electric potential, $\rho=Q / \forall\left(\mathrm{C} / \mathrm{m}^{3}\right)$ is the space charge density produced by corona electrode, $Q(\mathrm{C})$ is the amount of charge in a volume $\forall\left(\mathrm{m}^{3}\right), \varepsilon_{0}$ is the dielectric permittivity of free space equal to $8.854 \times 10^{-12}(\mathrm{~F} / \mathrm{m}), J\left(\mathrm{~A} / \mathrm{m}^{2}\right)$ is the current density, and $S\left(\mathrm{C} / \mathrm{m}^{3} . \mathrm{s}\right)$ is the source term for electric charge (electron and ion generation). When ions are generated in the vicinity of corona electrode, they can be transported through the medium by four mechanisms: convection, drift, mass diffusion, and charge diffusion. Therefore, the total ion flux is:

$$
J=\rho U+\rho Z_{i} E-D_{i}(\nabla \rho)+\sigma E .
$$

The four terms which appear in the derivation are the mathematical descriptions for these four mechanisms:

- First term: $\rho U$ is the convection (advection) current density and $U(\mathrm{~m} / \mathrm{s})$ is the velocity of the charge carriers;

- Second term: $\rho Z_{i} E=\rho U_{i}$ is ion drift current, where $U_{i}(\mathrm{~m} / \mathrm{s})$ is ion drift velocity and $Z_{i}\left(\mathrm{~m}^{2} / \mathrm{V} . \mathrm{s}\right)$ is ion mobility. $E(\mathrm{~V} / \mathrm{m})$ is the electric field intensity;

- Third term: $D_{i}(\nabla \rho)$ is the mass and charge diffusion current, where $D_{i}\left(\mathrm{~m}^{2} / \mathrm{s}\right)$ is the ion diffusivity;

- Fourth term: $\sigma E$ is the conduction current or charge diffusion (negligible), and $\sigma$ (A/V.m) is conductivity. 
The term of diffusion is usually negligible. Also, convection term can be ignored because the bulk velocity $U$ of the medium is much smaller (about two orders of magnitude) than $Z_{i} E$ in corona chargers. Consequently, $J$ with very good approximations becomes:

$$
J=\rho Z_{i} E .
$$

It is believed that a single-species model of the corona discharge is capable of capturing the underlying physics of steady-state corona for the study of charging process in the aerosol chargers. According to Kaptzov's assumption, when corona onsets with increasing the applied potential difference to the electrodes, the electric field at the surface of the ionization layer near the corona electrode will remain constant [13]. Ionization layer is very narrow near the corona electrode compared to the drift region and it is usually neglected in the simplified models and simulations. Thus, it can be considered that the electric field on the surface of the corona electrode will remain constant. The value of this constant $\left(E_{0}(\mathrm{kV} / \mathrm{m})\right)$ for wire and cylinder configurations is given by Peek's formula [14]:

$$
E_{0}=3300+\frac{79.53}{r_{0}}
$$

where $r_{0}(\mathrm{~m})$ is radius of the wire.

Eventually, Eq. (2) recasts to $\rho \nabla \cdot E+E \nabla \cdot \rho=0$ and considering $E=\nabla . V$, the following set of coupled equations is obtained which describes ion production in the charger of this study (which is decoupled from fluid flow equations).

$$
\left\{\begin{array}{l}
\nabla^{2} V=-\frac{\rho}{\varepsilon_{0}} \\
\nabla . J=\nabla V . \nabla \rho-\frac{\rho^{2}}{\varepsilon_{0}}=0
\end{array}\right.
$$

The second equation in Eq. (6) is called current continuity equation.

\subsubsection{Semi-analytic solution of $1 D$ corona discharge}

Far from the ends, the charger can be approximated with one-dimensional radial assumption. Therefore, the set of two equations in Eq. (6) is combined and reformed into the following non-linear third-order ODE:

$$
V^{\prime \prime \prime} V^{\prime}+V^{\prime \prime 2}+3 \frac{V^{\prime} V^{\prime \prime}}{r}=0
$$

The above relation describes electric potential variations along with the radial distance from corona wire in one-dimensional (axisymmetric) steady-state corona discharge. To find the voltage $V_{(r)}$ with respect to radial distance $r$ from the corona wire and three constants of integration, $c_{1}, c_{2}$, and $c_{3}$, few changes of variables were applied, which are listed in Table 1.

Consequently, electric potential, $V_{(r)}$, electric field, $E_{(r)}$, and space charge density, $\rho_{(r)}$, are given by the following relations:

$$
\begin{aligned}
& V_{(r)}=c_{1}\left[\sqrt{1-c_{2} r^{2}}-\ln \left(\sqrt{1-c_{2} r^{2}}+1\right)+\ln (r)\right]+c_{3}, \\
& E_{(r)}=-\nabla V=-\frac{c_{1} \sqrt{1-c_{2} r^{2}}}{r} \\
& \rho_{(r)}=\varepsilon_{0} c_{1} c_{2}\left(1-c_{2} r^{2}\right)^{-\frac{1}{2}}
\end{aligned}
$$

\begin{tabular}{|c|c|c|c|}
\hline \multicolumn{4}{|c|}{ Targeted O.D.E, $V^{\prime \prime \prime} V^{\prime}+V^{\prime \prime 2}+3 \frac{V^{\prime} V^{\prime \prime}}{r}=0$} \\
\hline Step & Change of variables & Differential equation & Type \\
\hline 1 & $\frac{d V_{(r)}}{d r}=F_{(r)}$ & $F F^{\prime}+F^{\prime 2}+3 \frac{F F^{\prime}}{r}=0$ & Homogenous 2nd Order \\
\hline 2 & $F_{(r)}=\exp \left(U_{(r)}\right)$ & $2 r U^{\prime 2}+r U^{\prime \prime}+3 U^{\prime}=0$ & Homogenous 2nd Order \\
\hline 3 & $\frac{d U_{(r)}}{d r}=W_{(r)}$ & $2 r W^{2}+3 W+r W^{\prime}=0$ & Bernoulli \\
\hline 4 & $Z_{(r)}=W_{(r)}^{-1}$ & $Z^{\prime}-3 \frac{Z}{r}-2=0$ & Homogenous 1st Order \\
\hline Step & Operation & \multicolumn{2}{|c|}{ Function } \\
\hline 5 & Solve for $Z$ & \multicolumn{2}{|c|}{$Z=r\left(c_{1} r^{2}-1\right)$} \\
\hline 6 & Solve for $W$ & \multicolumn{2}{|c|}{$W=\left(-r-c_{1} r^{3}\right)^{-1}$} \\
\hline 7 & Solve for $U$ & \multicolumn{2}{|c|}{$U=\ln (r)+\frac{1}{2} \ln \left(c_{1} r^{2}+1\right)+c_{2}$} \\
\hline 8 & Solve for $F$ & \multicolumn{2}{|c|}{$F=c_{2}\left(\frac{\sqrt{c_{1} r^{2}+1}}{r}\right)$} \\
\hline
\end{tabular}

Table 1. Solution procedure for Eq. (7).

Solution $V_{(r)}=c_{1}\left[\sqrt{1-c_{2} r^{2}}-\ln \left(\sqrt{1-c_{2} r^{2}}+1\right)+\ln (r)\right]+c_{3}$ 
Considering $r_{i}$ as the radius of the corona electrode, $r_{o}$ as the radius of the cylinder, $V_{i}$ as the corona voltage, $V_{o}$ as the second electrode (cylinder) voltage, and $E_{i}$ as the electric field intensity on the corona wire, potential difference $\Delta V$ and $E_{i}$ were combined to form the following relation:

$$
\begin{aligned}
& \frac{\Delta V}{E_{i}}= \\
& \frac{-r_{i}\left[\sqrt{1-c_{2} r_{i}^{2}}-\sqrt{1-c_{2} r_{o}^{2}}+\ln \left(\frac{\sqrt{1-c_{2} r_{o}^{2}}+1}{\sqrt{1-c_{2} r_{i}^{2}}+1}\right)+\ln \left(\frac{r_{i}}{r_{o}}\right)\right]}{\sqrt{1-c_{2} r_{i}^{2}}}
\end{aligned}
$$

To extract $c_{2}$ (implicitly) from the above relation, a MATLAB code was developed. Once $c_{2}$ is obtained, $c_{1}$ will be found by Eq. (9) and $c_{3}$ will be found by Eq. (8). Therefore, electric potential, $V_{(r)}$, electric field, $E_{(r)}$, and space charge density, $\rho_{(r)}$, will be calculated.

\subsubsection{Governing air flow field equations}

Navier-Stokes equation, which is the conservation of linear momentum, and Continuity equation, which is the conservation of mass, can describe behavior of the fluid flow. The Continuity equation is:

$$
\nabla \cdot\left(\rho_{f} U\right)=-\frac{\partial \rho_{f}}{\partial t} .
$$

Here, $\rho_{f}\left(\mathrm{~kg} / \mathrm{m}^{3}\right)$ is the fluid density and $U(\mathrm{~m} / \mathrm{s})$ is the velocity of the fluid or the medium. For steady incompressible flow, the continuity equation becomes:

$$
\nabla \cdot U=0 .
$$

Navier-Stokes equation is:

$$
\nabla \cdot\left(\rho_{f} U U\right)=-\nabla P+\rho_{f} g+\mu \nabla^{2} U+F_{B} .
$$

In the above equation, $P(\mathrm{~Pa})$ is the local fluid pressure, $g\left(\mathrm{~m} / \mathrm{s}^{2}\right)$ the gravitational acceleration, $\mu(\mathrm{kg} / \mathrm{m} . \mathrm{s})$ the dynamic viscosity of fluid, and $F_{B}\left(\mathrm{~N} / \mathrm{m}^{3}\right)$ the body force exerted per unit volume of the fluid. In the chargers, this force is due to the presence of the space charge density $\rho\left(\mathrm{C} / \mathrm{m}^{3}\right)$ and the electric field intensity $E(\mathrm{~V} / \mathrm{m})$. The steady and incompressible Navier-Stokes equation without turbulence model is used in this study. For calculations of particle residence time in the charger, we neglected the electro static force term $F_{B}$ in Navier-Stokes equations because the electrohydrodynamic number [15] is two orders of magnitude smaller than the Reynolds number.

The time required for a particle $\left(d_{P}<300 \mathrm{~nm}\right.$ in standard conditions) in the air stream to reach $95 \%$ of its terminal velocity or velocity of air (relaxation time) is on the order of $10^{-7} \mathrm{~s}$. This means that with a very good approximation, the velocity profile of the particles is the velocity profile of the fluid flow and the path lines are the trajectories. This fact is the consequence of small diameters and masses of very fine particles.

\subsection{Numerical simulations of steady-state corona discharge}

2.3.1. Scalar transport equation

Despite the fact that Eq. (6) is a simple model for corona simulations, it cannot be handled by some available commercial codes such as FLUENT 6.3.26. Standard computational fluid dynamics codes usually handle equations which are of scalar transport form [16]:

$$
\begin{aligned}
& \frac{\partial v \psi_{k}}{\partial t}+\frac{\partial}{\partial x_{i}}\left[v u_{i} \psi_{k}-\Gamma_{k} \frac{\partial \psi_{k}}{\partial x_{i}}\right]=S_{\psi_{k}}, \\
& k=1,2,3, \ldots, K
\end{aligned}
$$

where $v$ is fluid density ( $\rho$ has previously been used for current density), $\psi_{k}$ an arbitrary User-Defined Scalar (UDS) for the scalar number $k, u_{i}$ the convective velocity, $\Gamma_{k}$ the diffusion coefficient, and $S_{\psi k}$ the source term for each of the $K$ equations. It is not possible to cast the electric current continuity equation (the second equation in Eq. (6)) to the form above.

\subsubsection{Recasting equations}

As described in Section 2.2., the set in Eq. (6) governs steady-state corona discharge. The proposed algorithm converts current continuity equation into a Laplace equation. Space charge density is updated using an iteration loop. Since current density $J$ is an irrotational vector field [12], it can be found from the gradient of a scalar function as $J=-\nabla \psi$, where $\psi$ is different than the electric potential, $V$. One can use Eq. (6) and irrotationality condition to write:

$$
\nabla . J=0 \stackrel{J=-\nabla \psi}{\Rightarrow} \nabla^{2} \psi=0 .
$$

In this work, Eqs. (1) and (2) are solved. They need proper boundary conditions, which are the subject of next section.

\subsubsection{General boundary conditions}

For solving Eq. (1), proper boundary conditions should be applied. For the electric potential, simple Dirichlet boundary conditions are applied on the corona electrode (wire) and the grounded electrode (cylinder). A zero Neumann boundary condition is applied for the insulating parts of the charger and also for the inlet and outlet of the aerosol flow.

Current continuity equation is not directly solved because it is not in standard scalar transport form. Instead, we solve the derived equation, i.e. Eq. (16). Therefore, corresponding boundary conditions must be derived, too. A constant value for space charge density on the corona wire obtained from the semi-analytic solution can be applied. For electric potential, the Neumann boundary condition (zero flux) is applied for the insulating parts, inlet and outlet flows. However, 
this equation is solved indirectly by solving the Laplace equation. To solve Eq. (16), boundary conditions for $\rho$ and $V$ must be combined to give boundary conditions for $\psi$. We use the following relations:

$$
J=-\rho Z_{i} \nabla V^{J=-\nabla \psi} \nabla \psi=\rho Z_{i} \nabla V .
$$

On any surface, one could write:

$$
\frac{\partial \psi}{\partial n}=\rho Z_{i} \frac{\partial V}{\partial n}
$$

where $n$ represents the direction normal to the surface of the boundary. According to Eq. (16), $\rho$ can be obtained by:

$$
\rho=\frac{|\nabla \psi|}{Z_{i}|E|}=\frac{|\nabla \psi|}{Z_{i}|\nabla V|} .
$$

This equation is used to obtain a new distribution for $\rho$ in every iteration.

\subsubsection{Example of the application of boundary conditions (two electrodes configuration)}

Since the wire has a very small diameter, the analytic solution of a wire in infinite medium is held close to it. Therefore, on the wire, we use semi-analytic solution for $\rho$ and $\mathrm{Eq}$. (5) for $E$ to find $C_{1}$ (boundary condition for $\psi$ according to Table 2). Boundary conditions are listed in Table 2.

Parameter $C_{1}$ fixes normal derivative of $\psi$. On the cylinder, the boundary condition for $\psi$ is to be computed as a part of solution. We use current estimate of $\rho$ to find $E$ (indeed $V$ ) and compute $C_{2}=\rho E Z$ on the boundary (normal derivative of $\psi$ ). Solution of the Laplace equation for $\psi$ will give a new distribution for $\rho$, which modifies $V$ and hence $E$. It is hoped that repeating the calculations would give a converged solution for both $V$ and $\psi$. Numerical experiments show that it is essential for the solution to converge to modify $C_{2}$ before its use in such a way that it ensures total charge continuity is satisfied.

\subsubsection{Numerical algorithm}

The proposed algorithm is illustrated in Figure 2. According to this figure, in the first step, initial values

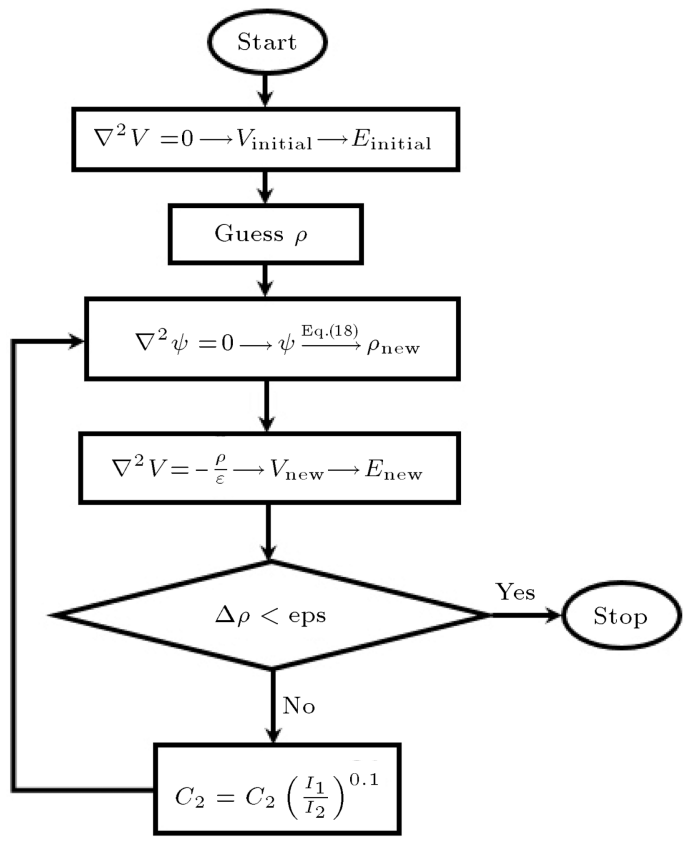

Figure 2. Flowchart of the simulation procedure.

of $E$ and $V$ are calculated considering value of $\rho$ to be zero. Then, initial guess for $\rho$ is used to compute $C_{2}$. Having $C_{2}$, the boundary conditions for $\psi$ are complete and Laplace equation for $\psi$ is solved to give new distribution of $\rho$. Now, Poisson's equation for $V$ can be solved with non-zero value of $\rho$ to obtain new values of $V$ and $E$. If convergence criterion on the change of $\rho$ is not satisfied, a new $C_{2}$ is computed based on the new $\rho$ and the procedure is repeated until convergence happens. In computing $C_{2}$, there is no guarantee that the total current is conserved. We found that correcting $C_{2}$ is essential for the stability of the method. To enforce total current conservation, we modify computed $C_{2}$ so that the ion current from the wire, $I_{1}$, is fully absorbed by the cylinder. We found by numerical experiments that modifying provisional value of $C_{2}$ with a factor of $\left(I_{1} / I_{2}\right)^{0.1}$ works well $\left(I_{2}\right.$ is the ionic current flowing to the cylinder). Using this boundary condition, new $\psi$ is found, which gives new $\rho$, and Poisson equation for $V$ is solved to give new $E$. The whole procedure is repeated until convergence happens.

Table 2. Applied boundary conditions. Note that there is no equation for $\rho$. Only physical boundary conditions are presented for $\rho$ to derive $\mathrm{BC}$ for $\psi$.

\begin{tabular}{cccc}
\hline Surface & $\begin{array}{c}\text { Electric } \\
\text { potential }\end{array}$ & $\begin{array}{c}\text { Space charge } \\
\text { density }\end{array}$ & $\boldsymbol{\psi}$ \\
\hline Wire (discharge electrode) & $V=V_{w}$ & $\rho=\rho_{c}$ & $\frac{\partial \psi}{\partial n}=\rho Z_{i} E=C_{1}$ \\
Cylinder & $V=V_{c}=0$ & $\frac{\partial \rho}{\partial n}=0$ & $\frac{\partial \psi}{\partial n}=\rho Z_{i} E=C_{2}$ \\
Insulating walls & $\frac{\partial V}{\partial n}=0$ & $\frac{\partial \rho}{\partial n}=0$ & $\frac{\partial \psi}{\partial n}=0$ \\
Inlet & $\frac{\partial V}{\partial n}=0$ & $\frac{\partial \rho}{\partial n}=0$ & $\frac{\partial \psi}{\partial n}=0$ \\
Outlet & $\frac{\partial V}{\partial n}=0$ & $\frac{\partial \rho}{\partial n}=0$ & $\frac{\partial \psi}{\partial n}=0$ \\
\hline
\end{tabular}




\subsubsection{Computation of space charge density in the hcarger}

If the perforated inner cylinder is conductor and is grounded as a third electrode, to solve Laplace equation for $\psi$, initial guess for $\rho$ is used to compute initial values of $C_{2}$ and $C_{3}$ (the boundary condition on perforated cylinder (third electrode) for $\psi$ ). In the final step of the algorithm, for stability of the method, the most recently computed values of $C_{2}$ and $C_{3}$ are modified so that the current from the wire $I_{1}$ is fully absorbed by two cylinders $\left(I_{1}=I_{2}+I_{3}\right)$ to enforce total current conservation. Numerical experiments showed that modifying provisional values of $C_{2}$ and $C_{3}$ with factors of $\left(I_{1} /\left(I_{2}+I_{3}\right)\right)^{A}$ and $\left(I_{1} /\left(I_{2}+I_{3}\right)\right)^{B}$ works well, where $A$ and $B$ are small experimentally found parameters.

\subsection{Estimation of induced charges}

Assuming that each molecule, on average, gains or loses one elementary charge (one electron), ion concentration $N_{\text {ion }}\left(\# / \mathrm{m}^{3}\right)$ can be calculated as:

$$
N_{\text {ion }}=\frac{\rho}{e}
$$

An approximation to the average number of charges induced on particles in the exposure of diffusion charging mechanism is given by White [17].

Charge distribution of monodisperse particles having diameter $d_{p}(\mathrm{~m})$ during time $t$ in the exposure of diffusion charging can be calculated by the following system of coupled first-order Ordinary Differential Equations (ODEs) Biskos [18]:

$$
\left\{\begin{array}{l}
\frac{d N_{0}}{d t}=\beta_{0} N_{0} N_{\text {ion }} \\
\frac{d N_{1}}{d t}=\beta_{0} N_{0} N_{\text {ion }}-\beta_{1} N_{1} N_{\text {ion }} \\
\vdots \\
\frac{d N_{n}}{d t}=\beta_{n-1} N_{n-1} N_{\text {ion }}-\beta_{n} N_{n} N_{\text {ion }}
\end{array}\right.
$$

where $N_{i}\left(\# / \mathrm{m}^{3}\right)$ ( $i$ runs from 0 to $n$ ) is the number concentration or fraction of particles having $i$ elementary charges; $n$ is the maximum number of induced charges on particles; and $\beta_{i}\left(\mathrm{~m}^{3} / \mathrm{s}\right)$ is the combination coefficients. Therefore, $N_{i}$ as functions of time will be found from $n+1$ relations and initial conditions in Eq. (22). Before entering the charger, particles are not charged. Therefore, initial conditions are $N_{0}=1$ and $N_{i}=0$ (for $i=1,2, \ldots, n$ ), which means percentages of uncharged particles and $i$ charged particles at the beginning of the process are $100 \%$ and $0 \%$, respectively.

For each particle diameter $d_{p}$, the set in Eq. (21) should be solved. We used standard ODE solver procedures to find charge distribution for each size class in a polydisperse aerosol.

\subsection{Aerodynamic simulations and optimizations}

The only information at hand about this charger was the schematic layout shown in Figure 1 and the size of the most important consisted parts. Therefore, according to this geometry, GAMBIT 2.2.30 was used to generate a fine mesh and introduced to FLUENT for aerodynamic simulation .

Vortices in the flow field are not desirable, causing aerosols to be lost or diluted. Particle residence times as a consequence of these vortices are non-uniform and therefore unacceptable. Hence, we rejected the original geometry because of the generated vortices and decided to modify it. The objective was to eliminate the vortices in the first geometry by streamlining the walls. We adapted a trial and error procedure to find a final geometry which removed the vortices by proposing and analyzing a new geometry step by step.

The ultimate grid for the final geometry is shown in Figure 3. It contains a fine triangular non-uniform mesh with nearly 100000 computational cells.

In Figure 4, path lines of released massless particles from the two inlets are illustrated and colored by particle ID. The particle loss decreases very much (approximately 6 particles crossed the perforated cylinder for every 100 released particles). Aerosol dilution is demonstrated here by air path lines passing through aerosol pathway.

As shown in Figure 5, path lines are colored by particle residence time. Ignoring the effect of boundary layers produced along the aerosol pathway, which are inevitable, all particles remain in the charger and are exposed to the bombardment of corona generated ions almost equally.

\section{Results and discussions}

The optimized geometry was the result of successive modifications of the geometry by streamlining the walls to eliminate vortices and reduce particle dilution and loss. This was managed by creating almost 30 new geometries, altering the geometry, and solving the flow field until no significant change was observed in the flow field.

The minimum residence time for 1000 particles passing the charging zone was 1.3 seconds and the maximum residence time was 31 seconds. The average residence time was 6.1 seconds and for a large number of particles, except for very few that travelled near the walls, was 2.2 seconds. This was computed without considering a small fraction of particles that appears near walls in very slow boundary layers. Although, because of the boundary layer effect, it is not possible to reach a design in which residence times of all particles are exactly equal, the accepted final design provided less deviant residence times from the mean. 


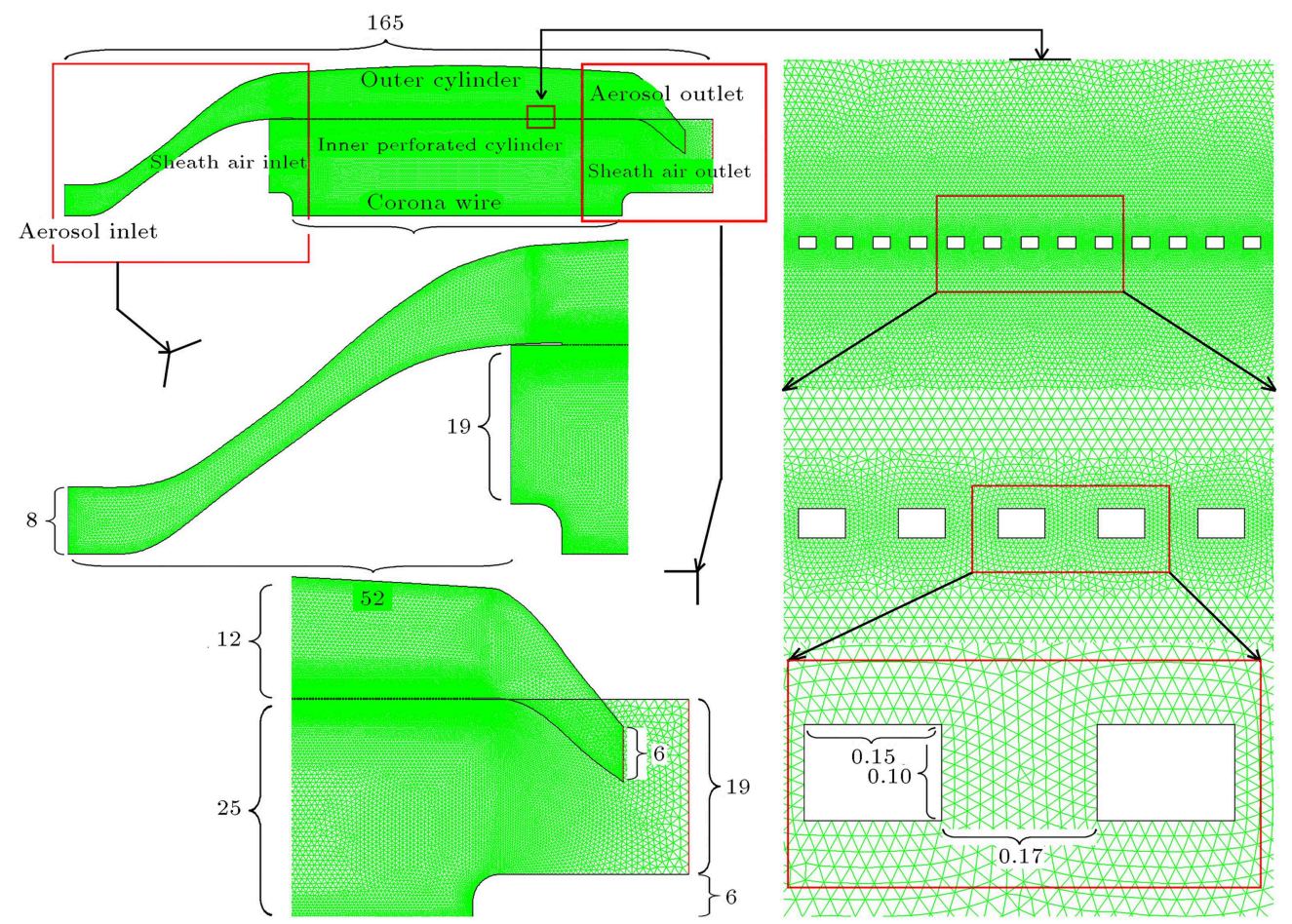

Figure 3. Generated grid for the final geometry and important dimensions in $\mathrm{mm}$.
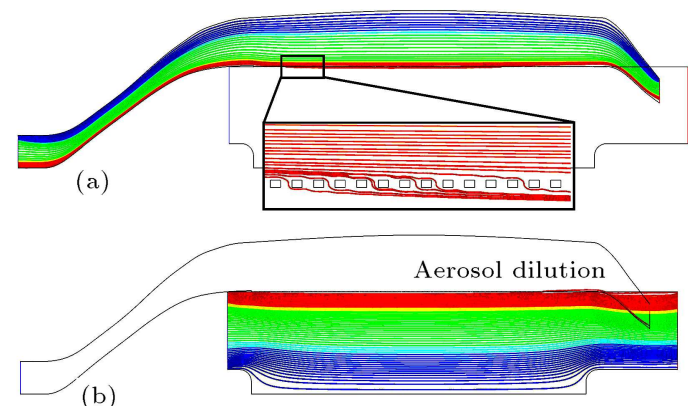

(b)

Figure 4. Aerosol loss (a) and dilution (b). Path lines are colored by particle ID for the final geometry.

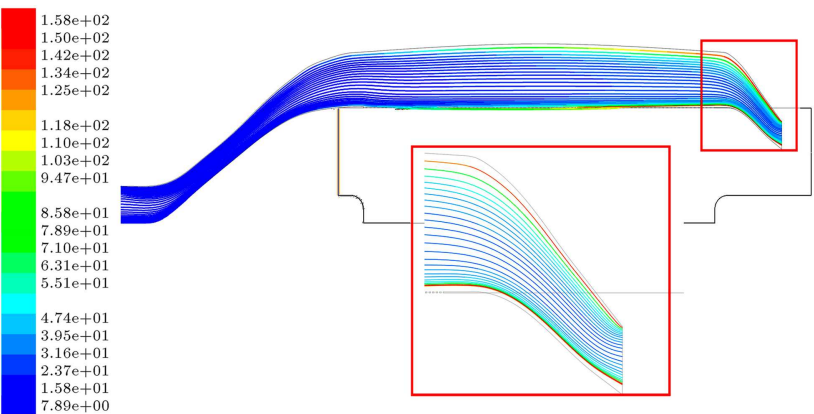

Figure 5. Particle traces colored by particle residence time $t(\mathrm{~s})$ for the final geometry.

To check validity of the proposed algorithm, a 2D axisymmetric problem of concentric wire and cylinder is solved and values of $\rho$ in the radial direction $r$ in the middle of the wire and cylinder are compared with results of the semi-analytic solution in Figure 6.

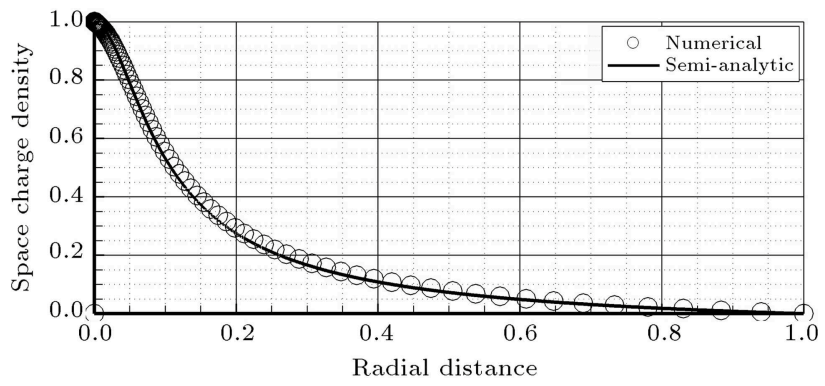

Figure 6. Comparison of the variations of $\rho$ along radial direction (normalized), resulted from the numerical simulation and semi-analytic solution.

The electrical simulations using the method described in Section 2 were carried out to find $\rho$ generated by high voltage corona wire. Results for radial variations of $N_{\text {ion }}$ in the two regions of the charger at the central cross section are demonstrated for different corona voltages $(5 \mathrm{kV}, 6 \mathrm{kV}$, and $7 \mathrm{kV}$ ) in Figure 7. Electric potential on the cylinders was set to zero for this simulations. This figure also compares the results with the results reported by Biskos et al. [18] in the voltage of $5 \mathrm{kV}$. Considering slight differences in the geometry of the chargers, both concentrations follow the same trend (they are not expected to match perfectly). The mismatch could be the result of the difference between sizes of the outer cylinders (due to the aerodynamic correction) and the semi-experimental method of obtaining space charge density by Biskos et al. [18] in contrast to this work. 


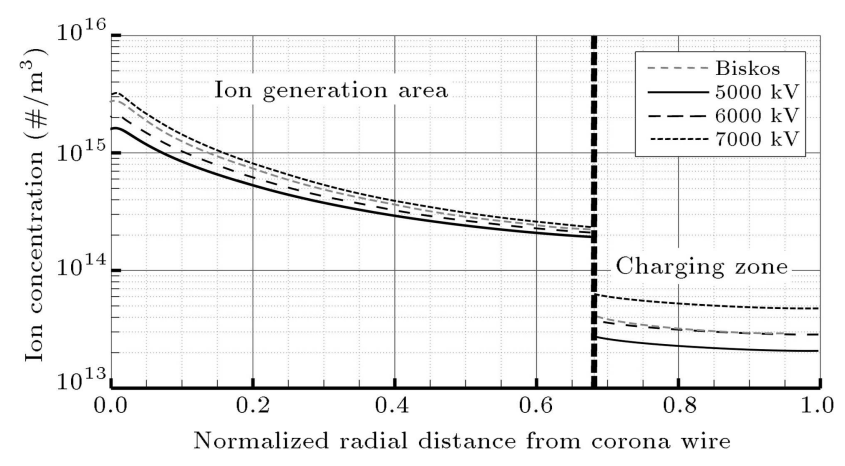

Figure 7. Radial variations of $N_{\text {ion }}$ in the two regions of the charger.

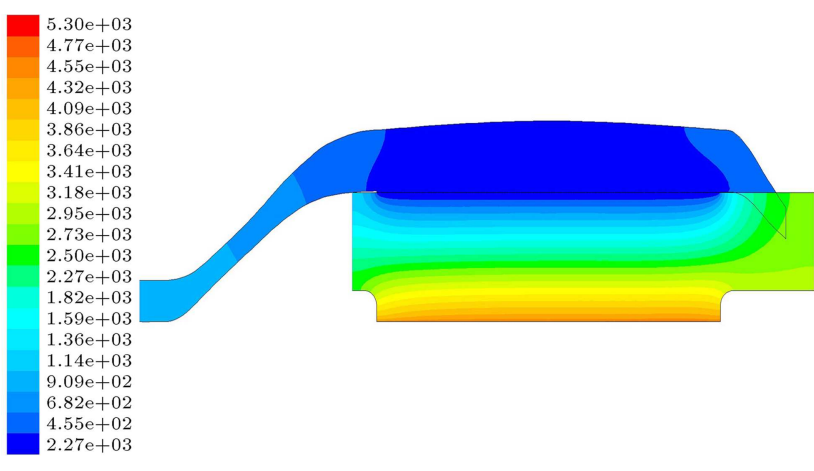

Figure 8. Electric potential contours.

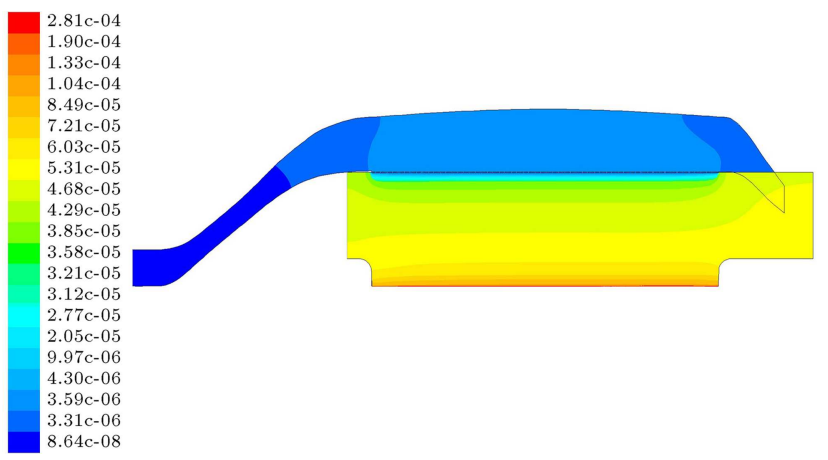

Figure 9. Space charge density contours. and 9 .

The contours of $V$ and $\rho$ are shown in Figures 8

Having ion concentration available, we classified polydisperse aerosols with appreciable concentrations between 100 and $300 \mathrm{~nm}$ into 11 size classes. Using 2.2 seconds of residence time, charge distribution was computed by solving Eq. (21). The result is given in Figure 10.

For the selected group of particles, the mean $N_{\text {ion }} \times t$ product was computed $2.3 \times 10^{13}\left(\# . \mathrm{s} / \mathrm{m}^{3}\right)$ and used for these calculations.

Figure 11 shows mean radial variations of $N_{\text {ion }} \times$ $t$ as functions of normalized radial distance $(L=$ 1) between two cylinders for three different corona voltages. For each radial distance corresponding to a particular streamline, the mean value of $N_{\text {ion }} \times t$

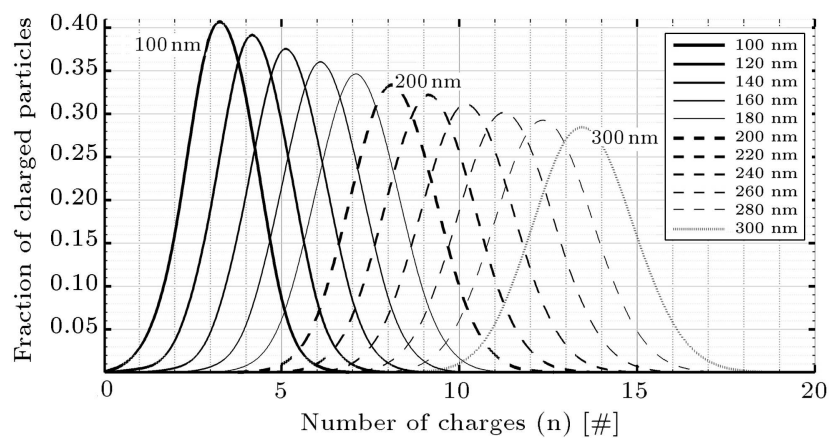

Figure 10. Distributions of charge on selected polydisperse particles.

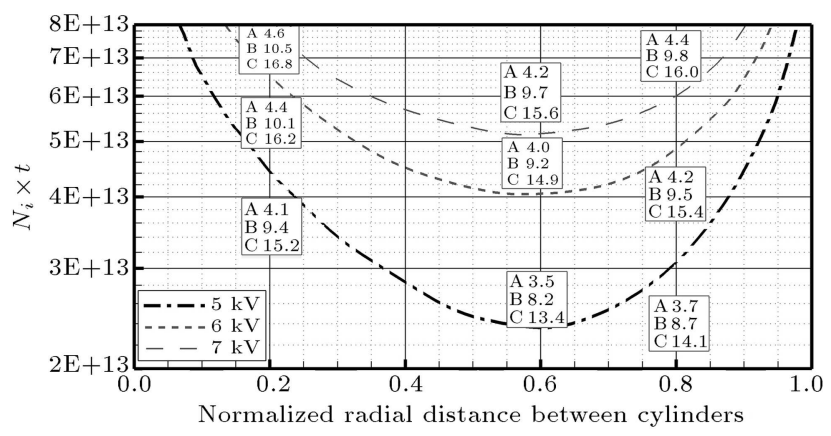

Figure 11. Radial variations of $N_{\text {ion }} \times t$ in the charging region for three different corona voltages. The boxes show average induced charge on $(A: 100 \mathrm{~nm}, B: 200 \mathrm{~nm}$, and $C: 300 \mathrm{~nm})$ particles.

was calculated by its integration along each streamline. Calculations of average charges for three diameters ( $A(100 \mathrm{~nm}), B(200 \mathrm{~nm})$, and $C(300 \mathrm{~nm}))$ were performed by their integration along each streamline in locations $0.2 L, 0.6 L$, and $0.8 L$. For the voltage of $5 \mathrm{kV}$, according to the figure, induced charges of 4.1 , 9.4 , and 15.2 were obtained, respectively, for 100-, 200, and 300-nanometer particles traveling in the vicinity of the boundary of the central cylinder $(0.2 L)$. For the particles traveling near the outer cylinder $(0.8 L)$, induced charges of $3.7,8.7$, and 14.1 were obtained and for the particles traveling in the middle $(\approx 0.6 L)$, the minimum charges of $3.5,8.2$, and 13.4 were calculated with respect to diameters $A, B$, and $C$. Other values of induced charge in the mentioned three locations were also obtained for corona voltages of $6 \mathrm{kV}$ and $7 \mathrm{kV}$, which were shown in the figure.

\section{Conclusion}

Aerosol characterizations using electrical techniques are more accurate with the aid of a well-designed charger in which particle residence times and the generated ion concentrations are more uniform and stable. Electrical simulations and aerodynamic corrections of the geometry of a wire-cylinder nanoparticle charger have been reported in this work. A 
new design was obtained by minimizing aerosol loss and dilution in the charger and eliminating vortices. This provided a more uniform exposure of particles to the ionic cloud bombardments. For the electrical simulations of the charger, a one-dimensional semianalytic solution of corona discharge was presented for estimating the ionic cloud productions. The more accurate estimation of ion concentrations was also carried out by proposing an algorithm for simulating corona discharge numerically on segregated solvers. The algorithm was coded in Fluent via UDF interface and tested against a simple corona configuration which lent itself to the semi-analytic solution. It was concluded that the proposed algorithm worked fine; thus, it can be used to model complex geometries where no analytic solution is expected. The obtained ion concentrations from numerical simulations of corona in the optimized charger were applied to find average charge and also charge distributions induced on polydisperse particles leaving the charger. The output of this solution, the charge distribution, could be used for particle tracing in EMS instruments and, therefore, finding size distribution from mobility distribution, or designing such instruments.

\section{Nomenclature}

$E_{i} \quad$ Electric field intensity on the wire $(\mathrm{V} / \mathrm{m})$

$\psi \quad$ Auxiliary parameter $\left(\mathrm{A} / \mathrm{m}^{4}\right)$

$\forall \quad$ Unit volume $\left(\mathrm{m}^{3}\right)$

$C_{1} \quad$ Normal derivative of $\psi$ (on the wire) $\left(\mathrm{A} / \mathrm{m}^{6}\right)$

$C_{2} \quad$ Normal derivative of $\psi$ (on the perforated cylinder $)\left(\mathrm{A} / \mathrm{m}^{6}\right)$

$C_{3} \quad$ Normal derivative of $\psi$ (on the outer cylinder $)\left(\mathrm{A} / \mathrm{m}^{6}\right)$

$D_{i} \quad$ Ion diffusivity $\left(\mathrm{m}^{2} / \mathrm{s}\right)$

$d_{p} \quad$ Diameter of particles $(\mathrm{m})$

$e \quad$ Single elementary charge equal to $1.6 \times 10^{-19} \mathrm{C}$

E $\quad$ Electric field intensity $(\mathrm{V} / \mathrm{m})$

$g \quad$ Gravitational acceleration $\left(\mathrm{m} / \mathrm{s}^{2}\right)$

$I_{1} \quad$ Ionic current from the wire $(\mathrm{A}, \mathrm{C} / \mathrm{s})$

$I_{2} \quad$ Ionic current flowing to the perforated cylinder $(\mathrm{C} / \mathrm{s})$

$I_{3} \quad$ Ionic current flowing to the outer cylinder $(\mathrm{C} / \mathrm{s})$

$J \quad$ Current density $\left(\mathrm{A} / \mathrm{m}^{2}\right)$

$\mu \quad$ Dynamic viscosity $(\mathrm{kg} / \mathrm{m} . \mathrm{s})$ $n$

Maximum number of induced charges on particles (\#)

$N_{i} \quad$ Number concentration (fraction) of particles achieved in $i$ units of charge $\left(\# / \mathrm{m}^{3}\right)$

$N_{\text {ion }} \quad$ Ion centration $\left(\# / \mathrm{m}^{3}\right)$

$P \quad$ Fluid pressure $(\mathrm{Pa})$

$Q \quad$ Amount of charge (C)

$r \quad$ Radial distance from the corona wire (m)

$r_{i}$

Radius of the wire (corona electrode) (m)

$r_{o} \quad$ Radius of the cylinder (ground electrode) $(\mathrm{m})$

$S \quad$ Electric charge source term $\left(\mathrm{C} / \mathrm{m}^{3} . \mathrm{s}\right)$

$t \quad$ Time $(\mathrm{s})$

$U \quad$ Velocity of the charge carriers $(\mathrm{m} / \mathrm{s})$

$U_{i} \quad$ Ion drift velocity $(\mathrm{m} / \mathrm{s})$

$V \quad$ Electric potential (V)

$V_{c} \quad$ Cylinder voltage $(\mathrm{V})$

$V_{i} \quad$ Corona voltage $(\mathrm{V})$

$V_{o} \quad$ Voltage of the second electrode (cylinder) (V)

$V_{w} \quad$ Corona voltage $(\mathrm{V})$

$Z_{i} \quad$ Ion mobility $\left(\mathrm{m}^{2} / \mathrm{V} . \mathrm{s}\right)$

$\beta_{i} \quad$ Combination coefficients $\left(\mathrm{m}^{3} / \mathrm{s}\right)$

$\varepsilon_{0} \quad$ Dielectric permittivity of free space equal to $8.854 \times 10^{-12}(\mathrm{~F} / \mathrm{m})$

$\rho \quad$ Space charge density $\left(\mathrm{C} / \mathrm{m}^{3}\right)$

$\rho_{c} \quad$ Space charge density on the corona wire $\left(\mathrm{C} / \mathrm{m}^{3}\right)$

$\rho_{f} \quad$ Fluid density $\left(\mathrm{Kg} / \mathrm{m}^{3}\right)$

$\sigma \quad$ Conductivity (A/V.m)

\section{References}

1. Hinds, W.C., Aerosol Technology, John Wiley \& Sons, New York, USA (1999).

2. Baron, P.A., Aerosol Measurements Principles, Techniques and Applications, John Wiley \& Sons, New York, USA (2001).

3. Choi, Y.J. and Kim, S.S. "An improved method for charging submicron and nano particles with uniform charging performance", J. Aerosol Sci. Technol., 41, pp. 259-265 (2007).

4. Tammet, H., Mirme, A. and Tamm, E. "Electrical aerosol spectrometer of Tartu University", Atmos. Env. Res., 62(3), pp. 315-324 (2002). 
5. Li, L. and Chen, D.R. "Performance study of a DCcorona-based particle charger for charge conditioning", J. Aerosol Science, 42(2), pp. 87-99 (2011).

6. Qi, C., Chen, D.R. and Greenberg, P. "Performance study of a unipolar aerosol mini-charger for a personal nanoparticle sizer", J. Aerosol Science, 39, pp. 450-459 (2008).

7. Park, D., Kim, Y.H., Lee, S.G., Kim, C. Hyang, J. and Kim, Y.J. "Development and performance test of a micro machined unipolar charger for measurements of submicron aerosol particles having a log-normal size distribution", J. Aerosol Science, 41, pp. 490-500 (2010).

8. Kruis, F.E. and Fissan, H. "Nanoparticle charging in a twin Hewitt charger", Journal of Nanoparticle Research, 3, pp. 39-50 (2001).

9. Alisoy, H.Z., Alagoz, S., Alisoy, G.H. and Alagoz, B.B. "A numerical method for the analysis of polydisperse aerosol particles charging in a coaxial electrode system", Journal of Electrostatics, pp. 1-6 (2011). DOI: 10.1016/j.elstat.2011.11.004

10. Intra, P. and Tippayawong, N. "Progress in unipolar corona dischargers design for airborne particle charging: A literature review", Journal of Electrostatics, 67, pp. 605-615 (2009).

11. Biskos, G., Reavell, K. and Collings, N. "Electrostatic characterization of corona-wire aerosol chargers", Journal of Electrostatics, 63(1), pp. 69-82 (2005).

12. Cheng, D.K., Field and Wave Electromagnetics, 3rd Ed., Addison-Wesley, Boston, USA (1917).

13. Kaptzov, N.A. "Elektrischeskiye yavleniya $\mathrm{v}$ gazakh 1 vacuume", OGIZ, Moscow (1947).

14. Peek, F.W., Dielectric Phenomena in High Voltage Engineering, 3rd Edn., McGraw-Hill, New York, USA (1929).
15. IEEE-DEIS-EHD Technical Committee "Recommended international standard for dimensionless parameters used in electrohydrodynamics", IEEE Trans. on Dielectrics and Electrical Insul., 10(1), pp. 3-6 (2003).

16. FLUENT 6.3.26 User's Guide, www.fluent.com.

17. White, H.J. "Particle charging in electrostatic precipitation", Transactions of the American Institute of Electrical Engineers, 70(2), pp. 1186-1191 (1951).

18. Biskos, G. "Theoretical and experimental investigation of the differential mobility spectrometer", Ph.D. Thesis, University of Cambridge, U.K. (2004).

\section{Biographies}

Afshin Shaygani works as Freelance Researcher and Mechanical Engineer. His research interests include aerosol science, CFD, micro technology, and machine learning.

Mohammad Said Saidi is a Professor (tenured) of Mechanical Engineering at Sharif University of Technology. His research interests include, but are not limited to, CFD, micro and macro multiphase flows in human body, experimental design, and mathematical modeling of transport of nano and microscale aerosol particles.

Mahdi Sani is currently serving as Faculty Member at Sharif University of Technology, Kish International Campus. He has served as CFD Engineer at Pargas Iran Co and as Mechanical Engineer at Monenco Iran and SADRA Companies. His research interests include aerosol science, CFD, micro technology, and biotechnology. 